OPEN ACCESS

Edited by:

Christoph Reiners,

University Hospital Würzburg,

Germany

Reviewed by:

Francesco Frasca

University of Catania, Italy

Silvia Martina Ferrari,

University of Pisa, Italy

*Correspondence:

Yong Jiang

yjiang8888@hotmail.com

Bao-Qiang Wu

gif55171@163.com

Specialty section:

This article was submitted to

Thyroid Endocrinology,

a section of the journal

Frontiers in Endocrinology

Received: 19 April 2020 Accepted: 17 September 2020

Published: 08 October 2020

Citation:

Feng J-W, Ye J, Wu W-X, Pan H,

Qin A-C, Jiang $Y$ and Wu B-Q (2020)

Management of Clinically Solitary

Papillary Thyroid Carcinoma Patients

According to Risk-Scoring Model for

Contralateral Occult Carcinoma.

Front. Endocrinol. 11:553577.

doi: 10.3389/fendo.2020.553577

\section{Management of Clinically Solitary Papillary Thyroid Carcinoma Patients According to Risk-Scoring Model for Contralateral Occult Carcinoma}

\author{
Jia-Wei Feng ${ }^{1}$, Jing Ye ${ }^{1}$, Wan-Xiao $W^{1}{ }^{1}$, Hua Pan ${ }^{1}$, An-Cheng Qin $^{2}$, Yong Jiang ${ }^{1 *}$ \\ and Bao-Qiang $\mathrm{Wu}^{3 *}$ \\ ${ }^{1}$ Department of Thyroid Surgery, The Third Affiliated Hospital of Soochow University, Changzhou First People's Hospital, \\ Changzhou, China, ${ }^{2}$ Department of General Surgery, The Affiliated Suzhou Hospital of Nanjing Medical University, Suzhou \\ Municipal Hospital, Suzhou, China, ${ }^{3}$ Department of General Surgery, The Second People's Hospital of Changzhou affiliated \\ to Nanjing Medical University, Changzhou, China
}

Objective: The aim of this study was to investigate risk factors of occult carcinoma in clinically solitary papillary thyroid carcinoma (PTC) patients, and to put emphasis on the predictive value of risk-scoring model to determine the optimal scope of surgery

Methods: A total of 573 clinically solitary PTC patients who underwent total thyroidectomy (TT) from two hospitals were retrospectively analyzed. Clinicopathological features were collected, univariate and multivariate analyses were performed to determine risk factors of occult carcinoma. The Cox proportional hazards model was used to analyze the risk factors of recurrence. A scoring model was constructed according to independent risk factors of contralateral occult carcinoma.

Results: $19.2 \%$ of clinically solitary PTC patients had occult carcinoma, among which 3.7\% patients had ipsilateral occult carcinoma and $15.5 \%$ patients had contralateral occult carcinoma. Factors such as male, the presence of benign nodule, and vascular invasion increase the risk of ipsilateral occult carcinoma. Tumor size $>1 \mathrm{~cm}$, the presence of benign nodule, extrathyroidal extension, central lymph node metastasis, lateral lymph node metastasis are independent predictors of contralateral occult carcinoma. Contralateral occult carcinoma is the independent predictor of recurrence. A 10-point risk-scoring model was established to predict the contralateral occult carcinoma in clinically solitary PTC patients.

Conclusion: Lobectomy is sufficient for clinically solitary PTC patients with risk factors of ipsilateral occult carcinoma. For clinically solitary PTC patients with score $\geq 4$, careful preoperative evaluations are required to rule out the contralateral occult carcinoma. Even if contralateral occult carcinoma is not detected preoperatively, $\Pi$ is recommended for high-risk patients.

Keywords: papillary thyroid microcarcinoma, contralateral occult carcinoma, ipsilateral occult carcinoma, recurrence-free survival, surgery 


\section{INTRODUCTION}

Papillary thyroid carcinoma (PTC) is the most common endocrine malignant tumor worldwide, accounting for approximately $80.0 \%$ of thyroid malignant tumors $(1,2)$. Although the incidence of PTC has increased rapidly in incidence, understanding of PTC has become more and more thorough from the molecular, diagnostic, and prognostic perspectives. According to the 2004 World Health Organization "Thyroid and Parathyroid Diseases and Genetic Classification", in addition to the most common classic PTC, there are 15 subtypes of PTC, among which highly aggressive PTC variants, such as diffuse sclerosing, tall-cell, insular, and poorly differentiated subtypes, exhibit heterogeneous clinical behavior and a wide range of mortality risk (3). Fine needle aspiration (FNA) of thyroid nodules can detect different gene mutations and other molecular changes in PTC, and the use of molecular pathways has permitted the development of new targeted therapies for aggressive PTC (4).

Despite the progress has been made in understanding the basic biological characteristics of PTC, the optimal scope of surgery, lobectomy vs total thyroidectomy (TT), is still controversial. As reported, PTC frequently occurs as multifocal lesions, with the prevalence of multifocality ranging from 20.0 to $36.1 \%(5-7)$. For tumors preoperatively detected in the bilateral lobes, there is no controversial to perform TT. Considering the possibility of recurrent or persistent carcinoma in the remnant contralateral lobe, TT seems to be applicable for tumors confined to the unilateral lobe. The rate of contralateral occult PTC discovered by TT was reported to range from 10 to $30 \%$ (811). Considering the high incidence of postoperative complications, the routine use of TT is not recommended for all patients with unilateral PTC. However, multifocal PTCs were reported to be associated with poor outcome, and increased risk of recurrence $(7,12,13)$. High resolution of ultrasonography (US), which could detect foci as small as $2-3 \mathrm{~mm}$, is currently used in detecting PTC and determining the scope of surgery (14). However, smaller tumors remain undetected. In addition, the ability of US to detect small malignant tumors would be significantly reduced when patients had diffuse thyroid disease (15).

Therefore, identifying risk factors of occult carcinoma in the thyroid lobe may help surgeons determine the optimal scope of surgery. By using a large series of patients who underwent initially TT for the treatment of a single PTC confined to only the unilateral lobe, we aimed to identify risk factors of occult carcinoma. In addition, we investigated the effects of occult carcinoma on clinical outcomes of patients. Different from previous studies, we established a model based on the risk

Abbreviations: PTC, papillary thyroid carcinoma; TT, total thyroidectomy; US, ultrasonography; FNA, fine-needle aspiration; CT, computed tomography; CND, central neck dissection; LND, lateral neck dissection; pCND, prophylactic central neck dissection; CLNM, central lymph node metastasis; cN0, clinically lymph node-negative; PTMC, papillary thyroid microcarcinoma; ETE, extrathyroidal extension; RFS, recurrence-free survival; LT4, levothyroxine; TSH, thyroidstimulating hormone; SD, standard deviations; ROC, receiver operating characteristic; ATA, American Thyroid Association. factors of occult carcinoma. According to this risk-scoring model, we can predict high-risk populations of occult carcinoma among solitary PTC patients for the avoidance of unnecessary TT, and provide individualized treatment for highrisk populations of occult carcinoma.

\section{MATERIALS AND METHODS}

\section{Patients}

This multi-center retrospective cohort study consisted of patients from Changzhou First People's Hospital and Suzhou Municipal Hospital between January 2011 and January 2018. These patients underwent initially TT for the treatment of a single PTC confined to only the unilateral lobe, without other suspicious carcinoma lesions in the contralateral lobe by preoperative image examinations or FNA. The Institutional Review Board of Changzhou First People's Hospital and Suzhou Municipal Hospital approved this retrospective study. All participants gave written informed consent for their clinical records to be used in this study. Patients were excluded from the study if they have any of following factors: (1) patients with preoperative clinical evidence of multiple PTCs or other pathologic types of thyroid malignancies; (2) patients with the single PTC had another suspicious lesion, but FNA was not performed for the suspicious lesion; (3) nonPTCs (medullary/follicular/anaplastic) or other subtypes than classic PTC (such as mixed PTC and so on); (4) patients did not undergo the TT; (5) patients had another malignancy before thyroidectomy; (6) patients with diffuse thyroid disease; (7) reoperation; (8) distant metastasis at diagnosis on pathological or clinical analysis; (9) history of neck radiation or familial cancer; (10) incomplete clinical data or missing follow-up. Finally, a total of 573 patients were enrolled in this study.

\section{Surgical Procedures}

We used the US, computed tomography (CT), or FNA to evaluate primary lesions and lymph nodes in the neck preoperatively. All patients underwent TT. TT was defined as the removal of two lobes, the isthmus, and the pyramidal lobe. For patients with clinically positive central lymph node metastasis (CLNM), therapeutic central neck dissection (CND) would be performed. All clinically lymph node-negative ( $\mathrm{cNO}$ ) PTC patients underwent prophylactic central neck dissection (pCND). CND plus therapeutic ipsilateral lateral neck dissection (LND) were performed for patients with clinically positive or intraoperative suspected lateral lymph node metastasis (LLNM). The central compartment refers to level VI. CND and pCND included the removal of prelaryngeal, pretracheal, and bilateral paratracheal lymph nodes (16). LND was performed in the usual fashion from at least level II to level V, sparing the internal jugular vein, spinal accessory nerve, and sternocleidomastoid muscle (17). According to neck levels, all lymph nodes specimens were separated by the surgeon and were sent to the department of pathology for paraffin fixation and histological analysis. 


\section{Histopathologic Examination of Surgical Specimens}

Two or more experienced pathologists reviewed and crosschecked all pathology specimens microscopically. Contralateral occult carcinoma, the tumor lesion detected by pathology postoperatively in the contralateral lobe rather than detected by preoperative examinations. Similarly, ipsilateral occult carcinoma was the tumor lesion detected by pathology postoperatively in the ipsilateral lobe rather than detected by preoperative examinations. Two or more PTC foci within the thyroid was defined as multifocality. Two or more PTC foci in a single lobe were ipsilateral multifocality, while two or more PTC foci in both lobes were bilateral multifocality. Papillary thyroid microcarcinoma (PTMC) was defined as PTC $\leq 1 \mathrm{~cm}$ in its maximum diameter. Extrathyroidal extension (ETE) was defined as the primary tumor extending through the thyroid capsule to perithyroidal soft tissue, or involving strap muscles, or extending to surrounding structures (18). PTC was subdivided into the following three groups according to the position: upper pole (upper part of the high plane of the isthmus), middle pole (parallel to the isthmus), and lower pole (lower part of the low plane of the isthmus). The location of the tumor was determined by the largest dominant lesion when the patient had multifocal lesions. When the dominant lesion occupied 2 adjacent parts, the location of the tumor was determined by the portion containing more than two-thirds of the tumor volume. Recurrence was defined as the any new lesions detected in cervical lymph nodes, or other organs on cytology from aspiration biopsy. Recurrencefree survival (RFS) was used to evaluate the outcomes. RFS was the duration started from the surgery to recurrence.

\section{Postoperative Management and Follow-Up}

Postoperative suppressive levothyroxine (LT4) treatment was conventionally performed in all patients. Thyroid-stimulating hormone (TSH) suppression therapy (serum TSH level below 0.5 $\mathrm{mIU} / \mathrm{L}$ ) with LT4 with or without radioactive iodine (RAI) ablation was administered to patients who underwent TT. After the initial surgery, physical examinations, US of the neck, and serum thyroid function (free thyroxin, TSH, thyroglobulin and anti-thyroglobulin antibodies) were performed for all patients every 6 months for a period of 2 years, and thereafter once a year. The criteria for remission was defined as (1) no evidence of tumor recurrence in clinical or radiologic examination, and (2) serum thyroglobulin levels of $<2 \mathrm{ng} / \mathrm{ml}$ during TSH stimulation and $<1 \mathrm{ng} / \mathrm{ml}$ during TSH suppression in the absence of anti-thyroglobulin antibodies. Disease recurrence, which included the local, regional, and distant recurrence, was defined as new evidence of pathologically proven recurrence in patients who initially met the criteria of remission. After the radiographic or biochemical examinations, histological examination of new lesion would be performed to verify whether the lesion was the recurrent PTC.

\section{Statistical Analyses}

All statistical analyses were performed using the SPSS v 25.0 software (Chicago, IL, USA). The continuous variables were expressed as means \pm standard deviations (SD). Univariate analysis for the comparison between patient groups was used Pearson's chi-square test or Fisher's exact test. Variables with a $P$ $<0.05$ in the univariate analysis were included in the multivariate analysis, which were performed logistic regression analysis to assess risk factors of contralateral/ipsilateral occult carcinoma. The potential relationship between clinicopathological variables and recurrence was used the Cox proportional hazards model to analyze. A risk-scoring model was constructed to calculate the probability of contralateral occult carcinoma on the basis of results in the multivariate analysis. The independent risk factors were selected as scoring items. According to the beta coefficient obtained from the logistic regression model, the score of each risk factor was weighted. To make the scoring model simple, all the beta coefficient divided the least one and then rounded to the nearest whole number. The total score for each patient represented the sum of scores for each risk factor. Receiver operating characteristic (ROC) curve was used to evaluate the predictive performance of the scoring model and find an appropriate cut-off point.

\section{RESULTS}

\section{Base Clinicopathological Characteristics of Patients}

The baseline clinicopathological characteristics of patients are summarized in Table 1. Among the 573 PTC patients, there were 134 men and 439 women with the mean age of $44.7 \pm 12.3$ years (range from 19 to 80 years). The mean BMI was $22.39 \pm 5.34 \mathrm{~kg} /$ $\mathrm{m}^{2}$ (range from 11.03 to $39.06 \mathrm{~kg} / \mathrm{m}^{2}$ ), and $208(36.3 \%$ ) patients were overweight. The diameter of the tumors ranged from 0.10 to $6.00 \mathrm{~cm}$ with the mean diameter of $1.20 \pm 0.87 \mathrm{~cm}$. Among 64 PTC patients who performed BRAF mutation analysis, $56(87.5 \%)$ patients had BRAF mutation positivity. Histopathological examination of specimens showed that one hundred and six (18.5\%) patients had benign thyroid nodules. Occult PTC foci were detected in the contralateral lobe in 89 (15.5\%) patients, and in the ipsilateral lobe in $21(3.7 \%)$ patients. The ETE and vascular invasion were detected in $84(14.7 \%)$ and $28(4.9 \%)$ patients, respectively. Tumor located in the upper portion of the thyroid gland was detected in 221 (38.6\%) patients, and tumor located in the middle/lower lobe of thyroid was detected in $352(61.4 \%)$ patients. CLNM only was present in 207 patients (36.1\%), both CLNM and LLNM were present in 64 patients $(11.2 \%)$, and LLNM only was present in 20 patients $(3.5 \%)$. The mean number of removed and metastatic lymph nodes in the central compartment was $6.0 \pm 4.6$ and $2.5 \pm$ 1.5, respectively. And the mean number of removed and metastatic lymph nodes in the lateral compartment was $18.3 \pm$ 11.5 and $5.0 \pm 4.8$, respectively.

\section{Clinicopathological Factors Associated With Contralateral Occult Carcinoma}

Among 89 patients with occult lesions in the contralateral gland, 31 patients had occult lesions in the upper portion of the thyroid 
TABLE 1 | Clinicopathological characteristics of 573 PTC patients.

\begin{tabular}{|c|c|}
\hline Clinicopathological characteristics & No. (\%) \\
\hline \multicolumn{2}{|l|}{ Sex } \\
\hline Male & $134(23.4 \%)$ \\
\hline Female & 439 (76.6\%) \\
\hline Age (Y), Mean \pm SD (range) & $44.7 \pm 12.3(19-80)$ \\
\hline$\geq 55$ & $113(19.7 \%)$ \\
\hline$<55$ & $460(80.3 \%)$ \\
\hline BMI (kg/m²), Mean \pm SD (range) & $22.39 \pm 5.34(11.03-39.06)$ \\
\hline Normal & $365(63.7 \%)$ \\
\hline Overweight & $208(36.3 \%)$ \\
\hline Maximum tumor size (cm), Mean \pm SD (range) & $1.20 \pm 0.87(0.10-6.00)$ \\
\hline$\leq 1$ & $283(49.4 \%)$ \\
\hline$>1$ & 290 (50.6\%) \\
\hline \multicolumn{2}{|l|}{ BRAF mutation* } \\
\hline Absence & $8(12.5 \%)$ \\
\hline Presence & $56(87.5 \%)$ \\
\hline \multicolumn{2}{|l|}{ With benign nodule } \\
\hline Absence & 467 (81.5\%) \\
\hline Presence & 106 (18.5\%) \\
\hline \multicolumn{2}{|l|}{ Occult carcinoma } \\
\hline Absence & $463(80.8 \%)$ \\
\hline Contralateral occult carcinoma & $89(15.5 \%)$ \\
\hline Ipsilateral occult carcinoma & $21(3.7 \%)$ \\
\hline \multicolumn{2}{|l|}{ ETE } \\
\hline Absence & 489 (85.3\%) \\
\hline Presence & $84(14.7 \%)$ \\
\hline \multicolumn{2}{|l|}{ Vascular invasion } \\
\hline Absence & $545(95.1 \%)$ \\
\hline Presence & 28 (4.9\%) \\
\hline \multicolumn{2}{|l|}{ Tumor location } \\
\hline Upper & $221(38.6 \%)$ \\
\hline Middle/Lower & 352 (61.4\%) \\
\hline \multicolumn{2}{|l|}{ LNM } \\
\hline Without LNM & $282(49.2 \%)$ \\
\hline CLNM only & 207 (36.1\%) \\
\hline LLNM only & 20 (3.5\%) \\
\hline CLNM and LLNM & $64(11.2 \%)$ \\
\hline No. of removed LNs in CC, Mean \pm SD (range) & $6.0 \pm 4.6(2-32)$ \\
\hline No. of removed LNs in LC, Mean \pm SD (range) & $18.3 \pm 11.5(5-51)$ \\
\hline No. of metastatic LNs in CC, Mean \pm SD (range) & $2.5 \pm 1.5(0-18)$ \\
\hline No. of metastatic LNs in LC, Mean \pm SD (range) & $5.0 \pm 4.8(3-22)$ \\
\hline Recurrence & $31(5.4 \%)$ \\
\hline LNs & $29(5.1 \%)$ \\
\hline Lung & $2(0.3 \%)$ \\
\hline
\end{tabular}

PTC, papillary thyroid carcinoma; $Y$, year; SD, standard deviation; ETE, extrathyroidal extension; LNM, lymph node metastasis; CLNM, central lymph node metastasis; LLNM, lateral lymph node metastasis; LN, lymph node; CC, central compartment; LC, lateral compartment.

${ }^{*} B R A F$ mutation analysis was started in 2017 and it was performed in 64 patients with PTC.

glands, and 58 patients had occult lesions in the middle/lower lobe poles of the glands. The mean size of the contralateral occult lesion was $0.27 \pm 0.19 \mathrm{~cm}$ (range $0.10-0.35 \mathrm{~cm}$ ). No contralateral occult lesions exhibited the ETE. In Table 2, contralateral occult carcinoma presented the significant association with tumor size, the presence of benign nodule, ETE, vascular invasion, CLNM, and LLNM by univariate analysis (all $P<0.05$ ). All of these factors were included in the multivariate analysis and showed that tumor size $>1 \mathrm{~cm}$ (OR: 2.280, 95\% CI: 1.111-4.680, $P=$ 0.025), the presence of benign nodule (OR: 7.361, 95\% CI: 3.67814.731, $P<0.001$ ), ETE (OR: 15.324, 95\% CI: 7.428-31.615, $P<$ 0.001), CLNM (OR: 4.125, 95\% CI: 1.914-8.891, $P<0.001$ ),
LLNM (OR: 6.983, 95\% CI: 3.492-13.966, $P<0.001$ ) remained independent predictors of contralateral occult carcinoma.

\section{Clinicopathological Factors Associated With Ipsilateral Occult Carcinoma}

Of 21 patients with occult lesions in the ipsilateral gland, 6 patients had occult lesions in the upper portion of the thyroid glands, and 15 patients had occult lesions in the middle/lower lobe poles of the glands. The mean size of the ipsilateral occult lesion was $0.24 \pm 0.16 \mathrm{~cm}$ (range $0.10-0.31 \mathrm{~cm}$ ). No ipsilateral occult lesions exhibited the ETE. As summarized in Table 3, univariate analysis revealed that male, the presence of benign nodule, ETE, and vascular invasion were significantly associated with the presence of ipsilateral occult carcinoma (all $P<0.05$ ). All of these factors were included in the multivariate analysis and revealed that male (OR: 45.286, 95\% CI: 4.819-425.574, $P=$ 0.001 ), the presence of benign nodule (OR: 9.858 , 95\% CI: 2.120 45.842, $P=0.004$ ), and vascular invasion (OR: $68.081,95 \% \mathrm{CI}$ : 7.440-662.304, $P<0.001)$ were independent predictive factors of ipsilateral occult carcinoma.

\section{Predictors of RFS}

Postoperative follow-up ranged from 7 to 89 months (average follow-up period: 32 months). During follow-up, 31 (5.4\%) patients developed recurrent disease, including 29 (5.1\%) patients had cervical lymph nodes recurrence and $2(0.3 \%)$ patients had lung recurrence.

Cox regression model in relation to RFS was conducted to determine variables which influenced recurrence. Our results showed tumor size, bilaterality, ETE, and CLNM were factors associated with recurrence (all $P<0.05$ ). Multivariate analyses showed tumor size $>1 \mathrm{~cm}$ (HR: 2.147, 95\% CI: 1.005-4.585, $P=$ 0.048 ), bilaterality (HR: 5.818, 95\% CI: $2.196-15.415, P<0.001$ ), ETE (HR: $2.447,95 \%$ CI: $1.033-5.793, P=0.042$ ), and CLNM (HR: 5.230, 95\% CI: 1.818-15.046, $P=0.002$ ) were independent risk predictors of recurrence, while other investigated variables had no significant influence on RFS (Table 4).

\section{Development of Risk-Scoring Model to Predict Contralateral Occult Carcinoma}

Considering that the bilaterality was the independent risk predictor of recurrence, we established the risk-scoring model to predict the contralateral occult carcinoma in solitary PTC patients. As shown in Table 5, based on the beta coefficient of the five independent risk factors (tumor size, the presence of benign nodule, ETE, CLNM, and LLNM) identified in the multivariate analysis of contralateral occult carcinoma, a 10-point riskscoring model was constructed.

According to the scoring model, the percentage of positive contralateral occult carcinoma ranged from 1.3 to $100.0 \%$ in order of total score in PTC patients (Table 6). A ROC curve of the risk-scoring model for contralateral occult carcinoma was plotted, and the area under the curve of the model for the prediction of contralateral occult carcinoma was 0.910 (95\% CI: $0.872-0.948, P<0.001$ ), indicating that the discriminative power of this model is acceptable (Figure 1). Moreover, a total score of 
TABLE 2 | Associations between clinicopathological characteristics and contralateral occult carcinoma in PTC patients.

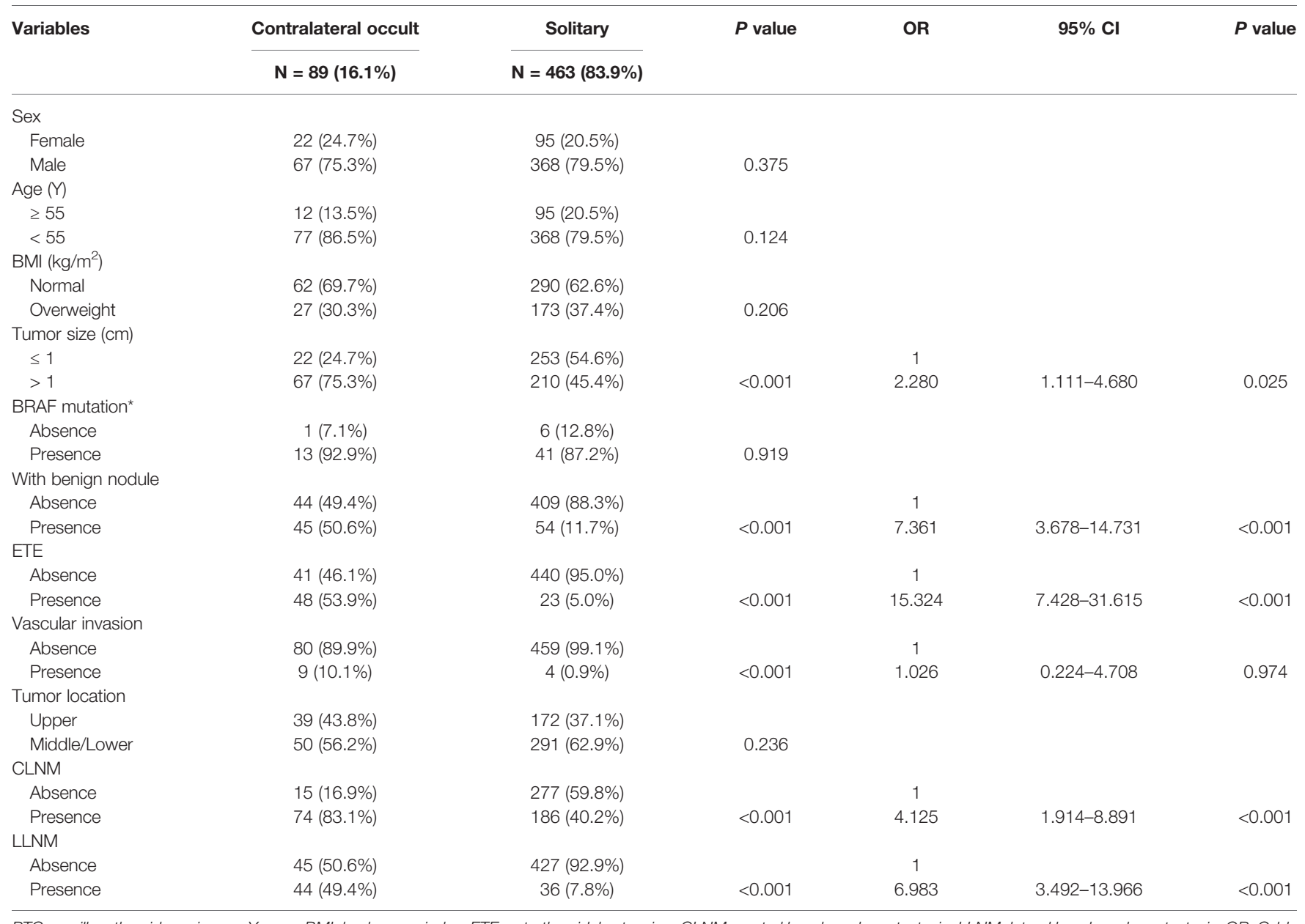

PTC, papillary thyroid carcinoma; Y, year; BMI, body mass index; ETE, extrathyroidal extension; CLNM, central lymph node metastasis; LLNM, lateral lymph node metastasis; OR, Odds ratio; $95 \% \mathrm{Cl}$, 95\% confidence interval.

*BRAF mutation analysis was started in 2017 and it was performed in 61 PTC patients in this table.

3.5 with the highest Youden's J value (0.717) was selected as the appropriate cut-off value for the model. Patients with a total score between 0 and 3 had the low risk of contralateral occult carcinoma, while patients with a total score ranging from 4 to 9 had the high risk of contralateral occult carcinoma.

\section{DISCUSSION}

Continued attention has been paid to PTC given its increasing incidence, which is reaching epidemic proportions. The treatment of PTC, especially PTMC, remains the controversial topic. According to the latest American Thyroid Association (ATA) guidelines, TT is recommended for patients with tumor size $>4 \mathrm{~cm}$, gross maximal ETE (T4), cervical lymph node metastasis, or distant metastasis. For preoperatively detected bilateral PTMC patients, TT is generally accepted, while for unilateral PTMC patients without ETE or cervical lymph node metastasis, lobectomy may be sufficient. In addition, considering that PTMC behaves more like a benign lesion, some scholars have proposed less aggressive strategies, such as "watch-and-wait" rather than surgery (19). However, some PTMCs may be occult and accompanied by aggressive behavior, which may lead to the local recurrence and cervical lymph node metastasis (8). In this study, we investigated the association between clinicopathological characteristics and occult carcinoma in order to identify solitary PTC patients with high risk of occult carcinoma, who would clearly benefit from a more extensive treatment.

In our study, we used the entire thyroid to determine the prevalence and distribution pattern of occult carcinoma in 573 consecutive patients with isolated nodule detected by preoperative US. Different from previous studies which defined "occult carcinoma" as the undetected carcinoma in the contralateral lobe when TT was performed in patients with preoperative unilateral PTC (20), we divided the occult carcinoma into contralateral and ipsilateral occult carcinoma. Our study found that $19.2 \%$ of clinically solitary PTC patients had occult carcinoma, among which $3.7 \%$ patients had ipsilateral occult carcinoma and $15.5 \%$ patients had contralateral occult carcinoma. The incidence of contralateral occult cancer in this study is consistent with the 10 to $30 \%$ reported in previous studies (8-11). Considering serious complications of TT, such as 
TABLE 3 | Associations between clinicopathological characteristics and ipsilateral occult carcinoma in PTC patients.

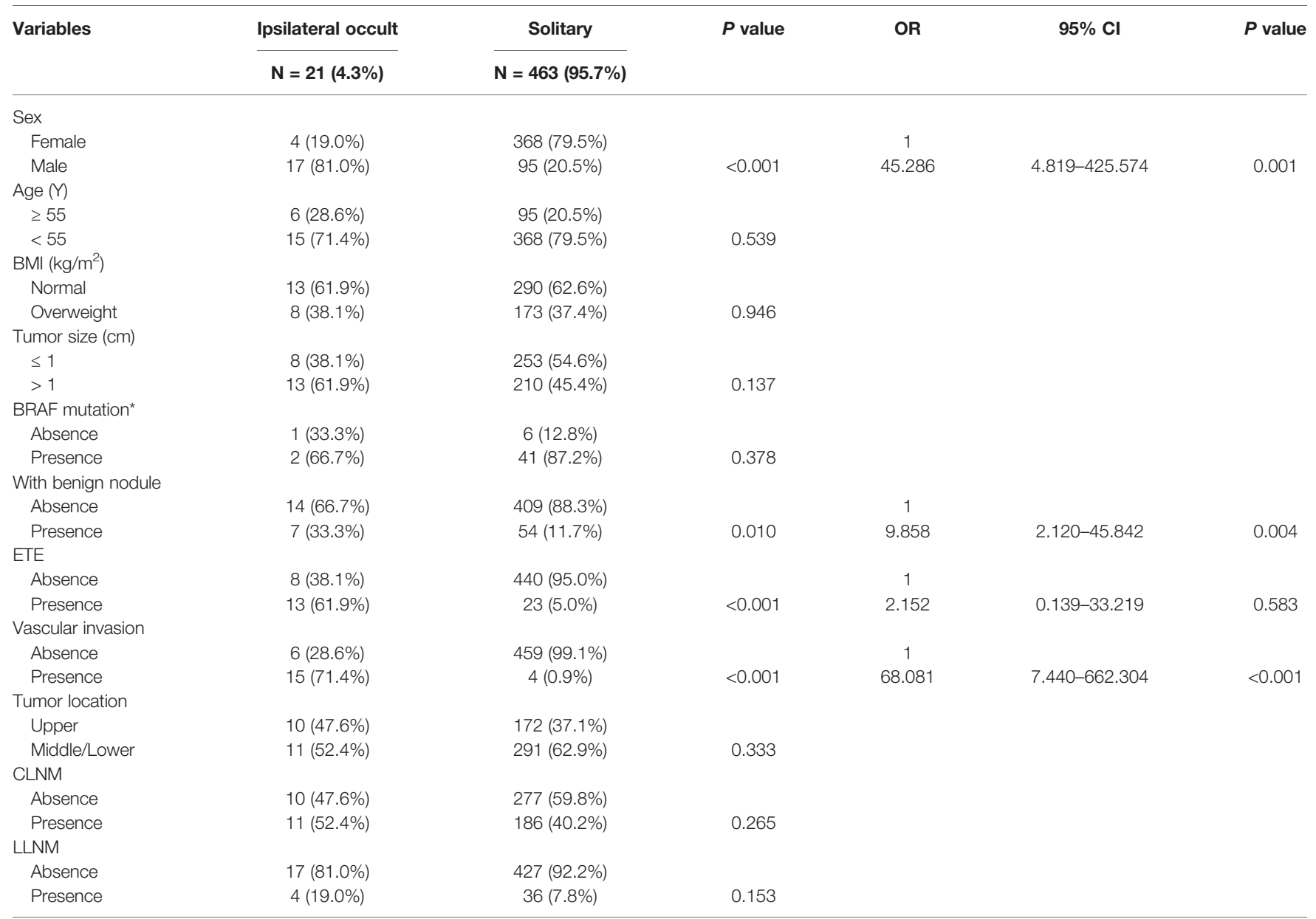

PTC, papillary thyroid carcinoma; Y, year; BMI, body mass index; ETE, extrathyroidal extension; CLNM, central lymph node metastasis; LLNM, lateral Iymph node metastasis; OR, Odds ratio; $95 \% \mathrm{Cl}$, 95\% confidence interval.

*BRAF mutation analysis was started in 2017 and it was performed in 50 PTC patients in this table.

vocal cord paralysis and hypoparathyroidism, the $15.5 \%$ incidence of contralateral occult carcinoma cannot convince all patients with clinically solitary PTC to routinely undergo TT. Hence, we further examined any clinicopathological characteristics related to the presence of occult carcinoma, especially contralateral occult carcinoma, to help select high-risk patients with occult carcinoma.

In this study, factors such as male, the presence of benign nodule, and vascular invasion were independent predictors of ipsilateral occult carcinoma. As for contralateral occult carcinoma, tumor size $>1 \mathrm{~cm}$, the presence of benign nodule, ETE, CLNM, LLNM were independent predictors. Our results were consistent with previous studies regarding the risk factors of multifocality (21-23). To our surprise, the presence of benign nodule was the risk factor of both contralateral and ipsilateral occult carcinoma. This result may be due to the presence of occult small carcinoma obscured by benign nodules. Moreover, the accurate of US in detecting lesions with diameters less than $5 \mathrm{~mm}$ was only $53.8 \%$ (24), and 7.9 to $18 \%$ of the aspirates were classified as "indeterminate" by FNA, which carry the risk of malignancy $(25,26)$. Therefore, the presence of benign nodule in the lobe by preoperative evaluation should be regarded as the significant predictive factor of potential presence of the occult carcinoma in the both contralateral and ipsilateral lobe.

Due to the excellent prognosis of PTMC, some people hold the view that the treatment of PTMC should be different from PTC (19). We further analyzed risk factors of recurrence. In addition to the tumor size $>1 \mathrm{~cm}$, ETE, and CLNM, which previously confirmed to be associated with recurrence (27-30), we found bilaterality, that is, the occult carcinoma in the contralateral lobe, is also the independent predictor of recurrence. However, ipsilateral multifocality, that is, the occult carcinoma in the ipsilateral lobe, has no association with recurrence. This result was consistent with previous studies. For example, a recent study involving 2,211 Chinese PTC patients showed the 10-year disease-free survival rate of patients with bilateral PTC was much lower than that of those with unilateral-multifocal and solitary PTC (78.8 vs 85.7 and $89.3 \%$, respectively; $P=0.005$ ) (31). Moreover, a study of 
TABLE 4 | Cox proportional hazards model demonstrating factors associated with recurrence-free survival in PTC patients.

\begin{tabular}{|c|c|c|c|c|c|c|}
\hline Variables & \multicolumn{3}{|c|}{ Univariate analyses } & \multicolumn{3}{|c|}{ Multivariate analyses ${ }^{*}$} \\
\hline \multicolumn{7}{|l|}{ Sex } \\
\hline Female & 1.652 & $0.706-3.865$ & 0.247 & & & \\
\hline \multicolumn{7}{|l|}{ Age $(Y)$} \\
\hline$<55$ & 1 & & & & & \\
\hline Normal & 1 & & & & & \\
\hline Overweight & 1.841 & $0.875-3.872$ & 0.108 & & & \\
\hline \multicolumn{7}{|l|}{ Tumor size $(\mathrm{cm})$} \\
\hline$\leq 1$ & 1 & & & 1 & & \\
\hline$>1$ & 2.621 & $1.094-6.280$ & 0.031 & 2.147 & $1.005-4.585$ & 0.048 \\
\hline \multicolumn{7}{|l|}{ Multifocality } \\
\hline \multicolumn{7}{|l|}{ ETE } \\
\hline Absence & 1 & & & 1 & & \\
\hline Presence & 2.560 & $1.040-6.299$ & 0.041 & 2.447 & $1.033-5.793$ & 0.042 \\
\hline \multicolumn{7}{|l|}{ Vascular invasion } \\
\hline Absence & 1 & & & & & \\
\hline Presence & 1.390 & $0.560-3.450$ & 0.477 & & & \\
\hline \multicolumn{7}{|l|}{ Tumor location } \\
\hline Middle/Lower & 1 & & & & & \\
\hline Upper & 1.322 & $0.659-2.650$ & 0.432 & & & \\
\hline \multicolumn{7}{|l|}{ CLNM } \\
\hline Absence & 1 & & & 1 & & \\
\hline Presence & 5.047 & $1.712-14.877$ & 0.003 & 5.230 & $1.818-15.046$ & 0.002 \\
\hline \multicolumn{7}{|l|}{ LLNM } \\
\hline Absence & 1 & & & & & \\
\hline
\end{tabular}

PTC, papillary thyroid carcinoma; Y, year; BMI, body mass index; ETE, extrathyroidal extension; CLNM, central lymph node metastasis; LLNM, lateral lymph node metastasis; HR, Hazard ratio; $95 \% \mathrm{Cl}$, 95\% confidence interval.

${ }^{*}$ The multivariate models were performed using a backward stepwise selection procedure. All clinically relevant variables with $P<0.05$ in univariate results were used in the multivariate model.

TABLE 5 | Development of a 10-point risk-scoring model to predict contralateral occult carcinoma in PTC patients.

\begin{tabular}{|c|c|c|c|}
\hline Variables & $P$ value & Beta coefficient & Point \\
\hline \multicolumn{4}{|c|}{$\begin{array}{l}\text { Tumor size }(\mathrm{cm}) \\
\leq 1\end{array}$} \\
\hline$>1$ & 0.025 & 0.824 & 1.000 \\
\hline \multicolumn{3}{|c|}{ With benign nodule } & \\
\hline Presence & $<0.001$ & 1.996 & 2.000 \\
\hline \multicolumn{4}{|l|}{ ETE } \\
\hline \multicolumn{4}{|l|}{ Absence } \\
\hline Presence & $<0.001$ & 2.729 & 3.000 \\
\hline \multicolumn{4}{|l|}{ CLNM } \\
\hline \multicolumn{4}{|l|}{ Absence } \\
\hline Presence & $<0.001$ & 1.417 & 2.000 \\
\hline \multicolumn{4}{|l|}{ LLNM } \\
\hline \multicolumn{4}{|l|}{ Absence } \\
\hline Presence & $<0.001$ & 1.944 & 2.000 \\
\hline
\end{tabular}

PTC, papillary thyroid carcinoma; CLNM, central lymph node metastasis; LLNM, lateral lymph node metastasis; ETE, extrathyroidal extension.

2,095 patients with PTC who underwent TT found that multifocality rather than bilaterality was the independent predictor of disease recurrence or persistence (32). Therefore,
TABLE 6 | Risk scores and percentage of contralateral occult carcinoma in PTC patients.

\begin{tabular}{lcccc}
\hline Risk score & $\begin{array}{c}\text { contralateral } \\
\text { occult } \\
\text { carcinoma } \\
(+)\end{array}$ & $\begin{array}{c}\text { contralateral } \\
\text { occult } \\
\text { carcinoma (-) }\end{array}$ & Total & $\begin{array}{c}\text { Positive } \\
\text { rate (\%) }\end{array}$ \\
\hline 0 & 2 & 147 & 149 & $1.3 \%$ \\
1 & 4 & 101 & 105 & $3.8 \%$ \\
2 & 1 & 74 & 75 & $1.3 \%$ \\
3 & 8 & 88 & 96 & $8.3 \%$ \\
4 & 8 & 18 & 26 & $30.8 \%$ \\
5 & 14 & 22 & 36 & $38.9 \%$ \\
6 & 25 & 11 & 36 & $69.4 \%$ \\
7 & 13 & 0 & 13 & $100.0 \%$ \\
8 & 7 & 2 & 9 & $77.8 \%$ \\
9 & 7 & 0 & 7 & $100.0 \%$ \\
\hline
\end{tabular}

PTC, papillary thyroid microcarcinoma.

conservative treatment, such as lobectomy, can be performed for clinically solitary PTC patients with risk factors of ipsilateral occult carcinoma.

In addition to showing contralateral occult carcinoma risk stratification data, we developed and validated a 10-point risk- 


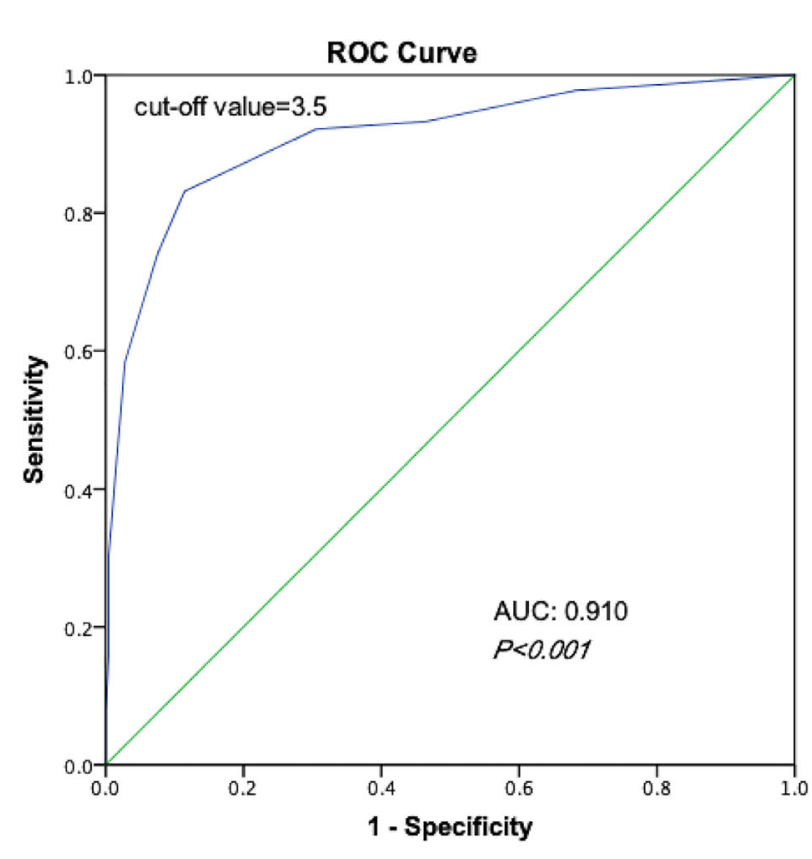

FIGURE 1 | Receiver operating characteristic curves of the ability of riskscoring model to predict the risk of contralateral occult carcinoma.

scoring model to predict the presence of contralateral occult carcinoma in clinically solitary PTC patients based on tumor size, the presence of benign nodule, ETE, CLNM, and LLNM in our study. Predictive models can not only understand the response probabilities of each predictor to other factors, but also quickly assess the response probabilities of individual subjects. According to the ROC curve, the area under the curve of the model for the prediction of contralateral occult carcinoma was 0.910 (95\% CI: $0.872-0.948, P<0.001$ ), showing the high discrimination accuracy of the scoring model. The average incidence of contralateral occult carcinoma in PTC patients with score $\geq 4$ (high risk) was significantly higher than that in patients with score $\leq 3$ (low risk) (58.3 vs. $3.5 \%, P<0.001$ ). The risk scoring model is simple and effective, which could be implemented in clinical work. By identifying the high-risk groups in the relatively objective manner, it can provide the personalized treatment for these patients. For example, combined with preoperative image examinations, clinically solitary PTC patients with score $\geq 4$ are recommended to undergo extensive treatment, such as $\mathrm{TT}$, to improve the prognosis, while clinically solitary PTC patients with score $\leq 3$ should undergo the lobectomy in consideration of high complication rates of TT

There are several potential limitations in our study, which expects to be extended upon in the further researches. First, our research was a retrospective study, which might have the selection bias. Compared with the prospective studies, retrospective studies tend to have more errors and biases. For instance, the data we provided were extracted from the document and were not captured in the actual conversation. The possibility of residual confounding variables of unmeasured factors could not be ruled out. Second, different surgeons were involved in performing TT and lymph node dissection. Postoperative results may be affected by surgeonspecific factors. Third, since LND is not generally recommended as a prophylactic procedure, there may be undetected LLNM. Moreover, the follow-up time was relatively short (average time: 32 months), which may lead to a low recurrence rate. Longer follow-up period may make the result more stable. Finally, more external validation, such as validation of the risk-scoring model in other institutions or other countries, is still necessary in the future.

In conclusion, our study found that male, the presence of benign nodule, and vascular invasion increase the risk of ipsilateral occult carcinoma in clinically solitary PTC patients. And tumor size $>1 \mathrm{~cm}$, the presence of benign nodule, ETE, CLNM, LLNM were independent predictors of contralateral occult carcinoma in clinically solitary PTC patients. Considering that bilaterality (occult carcinoma in the contralateral lobe) is the independent predictor of recurrence, we developed a 10-point riskscoring model for contralateral occult carcinoma to better guide the treatment in clinically solitary PTC patients. For clinically solitary PTC patients with score $\geq 4$, careful preoperative evaluations are required to exclude the contralateral occult carcinoma. Even if contralateral occult carcinoma is not detected preoperatively, TT is recommended for high-risk patients.

\section{DATA AVAILABILITY STATEMENT}

The raw data supporting the conclusions of this article will be made available by the authors, without undue reservation.

\section{ETHICS STATEMENT}

This study has been approved by the Institutional Review Board of Changzhou First People's Hospital and Suzhou Municipal Hospital ethics committee, and has been performed according to the ethical standards laid down in the 1964 Declaration of Helsinki.

\section{AUTHOR CONTRIBUTIONS}

A-CQ took charge of conceiving and designing the study. HP and $\mathrm{W}-\mathrm{XW}$ were responsible for collecting the data and analyzing and interpreting the data. J-WF took charge of writing the manuscript. JY was responsible for providing critical revisions. B-QW participated in the revision of this manuscript and put forward important revisions. Approving the final version of the manuscript was in charge of YJ. All authors contributed to the article and approved the submitted version.

\section{ACKNOWLEDGMENTS}

Lei Qin, the English language editor, was responsible for correcting language and grammar issues. 


\section{REFERENCES}

1. Seib CD, Sosa JA. Evolving Understanding of the Epidemiology of Thyroid Cancer. Endocrinol Metab Clin North Am (2019) 48(1):23-35. doi: 10.1016/ j.ecl.2018.10.002

2. Lim H, Devesa SS, Sosa JA, Check D, Kitahara CM. Trends in Thyroid Cancer Incidence and Mortality in the United States, 1974-2013. Jama (2017) 317 (13):1338-48. doi: 10.1001/jama.2017.2719

3. Ho AS, Luu M, Barrios L, Chen I, Melany M, Ali N, et al. Incidence and Mortality Risk Spectrum Across Aggressive Variants of Papillary Thyroid Carcinoma. JAMA Oncol (2020) 6(5):1-8. doi: 10.1001/jamaoncol.2019.6851

4. Ferrari SM, Fallahi P, Ruffilli I, Elia G, Ragusa F, Paparo SR, et al. Molecular testing in the diagnosis of differentiated thyroid carcinomas. Gland Surg (2018) 7(Suppl 1):S19-s29. doi: 10.21037/gs.2017.11.07

5. So YK, Kim MW, Son YI. Multifocality and bilaterality of papillary thyroid microcarcinoma. Clin Exp Otorhinolaryngol (2015) 8(2):174-8. doi: 10.3342/ ceo.2015.8.2.174

6. Ng SC, Kuo SF, Chen ST, Hsueh C, Huang BY, Lin JD. Therapeutic Outcomes of Patients with Multifocal Papillary Thyroid Microcarcinomas and Larger Tumors. Int J Endocrinol (2017) 2017:4208178. doi: 10.1155/2017/4208178

7. Kuo SF, Lin SF, Chao TC, Hsueh C, Lin KJ, Lin JD. Prognosis of multifocal papillary thyroid carcinoma. Int J Endocrinol (2013) 2013:809382. doi: $10.1155 / 2013 / 809382$

8. Chow SM, Law SC, Chan JK, Au SK, Yau S, Lau WH. Papillary microcarcinoma of the thyroid-Prognostic significance of lymph node metastasis and multifocality. Cancer (2003) 98(1):31-40. doi: 10.1002/ cncr.11442

9. Hay ID, Hutchinson ME, Gonzalez-Losada T, McIver B, Reinalda ME, Grant CS, et al. Papillary thyroid microcarcinoma: a study of 900 cases observed in a 60-year period. Surgery (2008) 144(6):980-987; discussion 987-988. doi: 10.1016/j.surg.2008.08.035

10. Mercante G, Frasoldati A, Pedroni C, Formisano D, Renna L, Piana S, et al. Prognostic factors affecting neck lymph node recurrence and distant metastasis in papillary microcarcinoma of the thyroid: results of a study in 445 patients. Thyroid (2009) 19(7):707-16. doi: 10.1089/thy.2008.0270

11. Schönberger J, Marienhagen J, Agha A, Rozeboom S, Bachmeier E, Schlitt H, et al. Papillary microcarcinoma and papillary cancer of the thyroid $<\mathrm{or}=1 \mathrm{~cm}$ : modified definition of the WHO and the therapeutic dilemma. Nuklearmedizin (2007) 46(4):115-20quiz N141-112. doi: 10.1160/nukmed0059

12. Khan M, Syed AA, Khan AI, Hussain SR, Urooj N. Association of tumor size and focality with recurrence/persistence in papillary thyroid cancer patients treated with total thyroidectomy along with radioactive-iodine ablation and TSH suppression. Updates Surg (2018) 70(1):121-7. doi: 10.1007/s13304-0170465-5

13. Choi WR, Roh JL, Gong G, Cho KJ, Choi SH, Nam SY, et al. Multifocality of papillary thyroid carcinoma as a risk factor for disease recurrence. Oral Oncol (2019) 94:106-10. doi: 10.1016/j.oraloncology.2019.05.023

14. Chen HY, Liu WY, Zhu H, Jiang DW, Wang DH, Chen Y, et al. Diagnostic value of contrast-enhanced ultrasound in papillary thyroid microcarcinoma. Exp Ther Med (2016) 11(5):1555-62. doi: 10.3892/etm.2016.3094

15. Lee J, Park JH, Lee CR, Chung WY, Park CS. Long-term outcomes of total thyroidectomy versus thyroid lobectomy for papillary thyroid microcarcinoma: comparative analysis after propensity score matching. Thyroid (2013) 23(11):1408-15. doi: 10.1089/thy.2012.0463

16. Carty SE, Cooper DS, Doherty GM, Duh QY, Kloos RT, Mandel SJ, et al. Consensus statement on the terminology and classification of central neck dissection for thyroid cancer. Thyroid (2009) 19(11):1153-8. doi: 10.1089/ thy.2009.0159

17. Stack BC, Ferris RL, Goldenberg D, Haymart M, Shaha A, Sheth S, et al. American Thyroid Association consensus review and statement regarding the anatomy, terminology, and rationale for lateral neck dissection in differentiated thyroid cancer. Thyroid (2012) 22(5):501. doi: 10.1089/thy.2011.0312

18. Tuttle RM, Haugen B, Perrier ND. Updated American Joint Committee on Cancer/Tumor-Node-Metastasis Staging System for Differentiated and
Anaplastic Thyroid Cancer (Eighth Edition): What Changed and Why? Thyroid (2017) 27(6):751-6. doi: 10.1089/thy.2017.0102

19. D’Agostino TA, Shuk E, Maloney EK, Zeuren R, Tuttle RM, Bylund CL. Treatment decision making in early-stage papillary thyroid cancer. Psychooncology (2018) 27(1):61-8. doi: 10.1002/pon.4383

20. Pelizzo MR, Boschin IM, Toniato A, Piotto A, Bernante P, Pagetta C, et al. Papillary thyroid microcarcinoma (PTMC): prognostic factors, management and outcome in 403 patients. Eur J Surg Oncol (2006) 32(10):1144-8. doi: 10.1016/j.ejso.2006.07.001

21. Wang P, Wang Y, Miao C, Yu X, Yan H, Xie Q, et al. Defining a New Tumor Dimension in Staging of Papillary Thyroid Carcinoma. Ann Surg Oncol (2017) 24(6):1551-6. doi: 10.1245/s10434-017-5764-Z

22. Kiriakopoulos A, Petralias A, Linos D. Multifocal Versus Solitary Papillary Thyroid Carcinoma. World J Surg (2016) 40(9):2139-43. doi: 10.1007/s00268016-3628-5

23. Vorasubin N, Nguyen C, Wang M. Risk factors for cervical lymph node metastasis in papillary thyroid microcarcinoma: A meta-analysis. Ear Nose Throat J (2016) 95(2):73-7.

24. Ha SM, Kim JK, Baek JH. Detection of Malignancy Among Suspicious Thyroid Nodules $<1 \mathrm{~cm}$ on Ultrasound with Various Thyroid Image Reporting and Data Systems. Thyroid (2017) 27(10):1307-15. doi: 10.1089/ thy.2017.0034

25. Piana S, Frasoldati A, Ferrari M, Valcavi R, Froio E, Barbieri V, et al. Is a fivecategory reporting scheme for thyroid fine needle aspiration cytology accurate? Experience of over 18,000 FNAs reported at the same institution during 1998-2007. Cytopathology (2011) 22(3):164-73. doi: 10.1111/j.13652303.2010.00777.x

26. Nayar R, Ivanovic M. The indeterminate thyroid fine-needle aspiration: experience from an academic center using terminology similar to that proposed in the 2007 National Cancer Institute Thyroid Fine Needle Aspiration State of the Science Conference. Cancer (2009) 117(3):195-202. doi: 10.1002/cncy.20029

27. Hughes CJ, Shaha AR, Shah JP, Loree TR. Impact of lymph node metastasis in differentiated carcinoma of the thyroid: a matched-pair analysis. Head Neck (1996) 18(2):127-32. doi: 10.1002/(SICI) 1097-0347(199603/04)18:2<127:: AID-HED3>3.0.CO;2-3

28. Lee J, Song Y, Soh EY. Central lymph node metastasis is an important prognostic factor in patients with papillary thyroid microcarcinoma. J Korean Med Sci (2014) 29(1):48-52. doi: 10.3346/jkms.2014.29.1.48

29. Beasley NJ, Lee J, Eski S, Walfish P, Witterick I, Freeman JL. Impact of nodal metastases on prognosis in patients with well-differentiated thyroid cancer. Arch Otolaryngol Head Neck Surg (2002) 128(7):825-8. doi: 10.1001/ archotol.128.7.825

30. Lee KJ, Cho YJ, Kim SJ, Lee SC, Kim JG, Ahn CJ, et al. Analysis of the clinicopathologic features of papillary thyroid microcarcinoma based on 7 mm tumor size. World J Surg (2011) 35(2):318-23. doi: 10.1007/s00268-0100886-5

31. Wang W, Su X, He K, Wang Y, Wang H, Wang H, et al. Comparison of the clinicopathologic features and prognosis of bilateral versus unilateral multifocal papillary thyroid cancer: An updated study with more than 2000 consecutive patients. Cancer (2016) 122(2):198-206. doi: 10.1002/cncr.29689

32. Kim HJ, Sohn SY, Jang HW, Kim SW, Chung JH. Multifocality, but not bilaterality, is a predictor of disease recurrence/persistence of papillary thyroid carcinoma. World J Surg (2013) 37(2):376-84. doi: 10.1007/s00268-012-1835-2

Conflict of Interest: The authors declare that the research was conducted in the absence of any commercial or financial relationships that could be construed as a potential conflict of interest.

Copyright (C) 2020 Feng, Ye, Wu, Pan, Qin, Jiang and Wu. This is an open-access article distributed under the terms of the Creative Commons Attribution License (CC BY). The use, distribution or reproduction in other forums is permitted, provided the original author(s) and the copyright owner(s) are credited and that the original publication in this journal is cited, in accordance with accepted academic practice. No use, distribution or reproduction is permitted which does not comply with these terms. 